\title{
Real Hypersurfaces of Type A in Complex Two-Plane Grassmannians Related to The Reeb Vector Field
}

\author{
Ruenn-Huah Lee ${ }^{a}$ and Tee-How Loo ${ }^{b}$ \\ ${ }^{\mathrm{a}, \mathrm{b}}$ Institute of Mathematical Sciences, Faculty of Science \\ University of Malaya, 50603 Kuala Lumpur, Malaysia \\ rhlee063@siswa.um.edu.my,looth@um.edu.my
}

\begin{abstract}
Y. J. Suh and H. Lee (Bull. Korean. Math. Soc. 47, 551-561 (2010)) characterized real hypersurfaces $M$ of type $B$ by the invariance of vector bundle $J T M^{\perp}$ under the shape operator and the orthogonality of $J T M^{\perp}$ and $\mathrm{J}^{\perp} M^{\perp}$ , where $T M^{\perp}, J$ and $\mathrm{J}$ are the normal bundle of $M$, Kähler structure and Quaternionic Kähler structure of $G_{2}\left(C^{m+2}\right)$ respectively. In this paper, we characterize real hypersurfaces $M$ of type $A$ by the invariance of the vector bundle $J T M^{\perp}$ under the shape operator with the Reeb vector field in $\mathrm{J} T M^{\perp}$.
\end{abstract}

Keywords: complex Grassmannians; real hypersurfaces; tubes.

\section{INTRODUCTION}

The beauty of real hypersurfaces of complex two-plane Grassmannians was not discovered and fully appreciated until J. Berndt provided a thorough study on the Riemannian geometry of the complex two-plane Grassmannians [1]. The complex two-plane grassmannian has some remarkable properties and structures. The most notable one being the fact that it is the unique compact irreducible Riemannian symmetric space with both a Kähler structure $J$ and a quaternionic Kähler structure $\mathbf{J}$. These geometric structures induce a local almost contact 3-structure $\left(\phi_{a}, \xi_{a}, \eta_{a}\right), a \in\{1,2,3\}$ as well as an almost contact structure $(\phi, \xi, \eta)$ on its real hypersurfaces $M$.

The development of the theory of real hypersurfaces in complex two-plane Grassmannians has been at its prime after the characterization of real hypersurfaces of type $A$ and type $B$ given by J. Berndt and Y. J. Suh in the late 90s [2]. In the following, we denote by $D^{\perp}$, the space spanned by $\left\{\xi_{1}, \xi_{2}, \xi_{3}\right\}$ :

Theorem 1.1. ([2]) Let $M$ be a connected real hypersurface in $G_{2}\left(C^{m+2}\right), m \geq 3$. Then both $\xi$ and $\mathrm{D}^{\perp}$ are invariant under the shape operator of $M$ if and only if

(A) $M$ is an open part of a tube around a totally geodesic $G_{2}\left(C^{m+1}\right)$ of $G_{2}\left(C^{m+2}\right)$, or

(B) $m$ is even, say $m=2 n$, and $M$ is an open part of a tube around a totally geodesic $\mathrm{H} P^{n}$ in $G_{2}\left(C^{m+2}\right)$.

We say that a real hypersurface $M$ in $G_{2}\left(C^{m+2}\right)$ is of type $A$ if it satisfies the first property in the characterization theorem given above. On the other hand, $M$ is said to be of type $B$ if it satisfies all properties in part (B). A connected orientable real hypersurface $M$ in $G_{2}\left(C^{m+2}\right)$ is said to be Hopf if the Reeb vector field $\xi$ is invariant under the shape operator of $M$. The following theorems were also given in the same paper, provide sufficient conditions of being a real hypersurface of type $A$ and type $B$.

Theorem 1.2. ([2]) Let $M$ be a connected real hypersurface of $G_{2}\left(C^{m+2}\right), m \geq 3$. If $A \mathrm{D} \subset \mathrm{D}, A \xi=\alpha \xi$ and $\xi$ is tangent to $\mathrm{D}^{\perp}$, then $M$ is an open part of a tube around a totally geodesic $G_{2}\left(C^{m+1}\right)$ in $G_{2}\left(C^{m+2}\right)$.

Theorem 1.3. ([2]) Let $M$ be a connected real hypersurface of $G_{2}\left(C^{m+2}\right), m \geq 3$. If $A \mathrm{D} \subset \mathrm{D}, A \xi=\alpha \xi$ and $\xi$ is tangent to $\mathrm{D}$, then $m=2 n$ and $M$ is an open part of a tube around a totally geodesic $\mathrm{H} P^{n}$ in $G_{2}\left(C^{m+2}\right)$. 
These characterizations of real hypersurfaces of type $A$ and type $B$ look extremely similar. Recently, H. Lee and Y. J. Suh shown that the invariance of $\mathrm{D}$ under $A$ is in fact a consequence of being a Hopf hypersurface with the Reeb vector field belongs to $D[3]$.

Theorem 1.4. ([3]) Let $M$ be a connected orientable Hopf hypersurface in $G_{2}\left(C^{m+2}\right), m \geq 3$. Then the Reeb vector $\xi$ belongs to the distribution $\mathrm{D}$ if and only if $M$ is locally congruent to an open part of a real hypersurface of type $B$.

The preceding theorem improves Theorem 1.3 significantly. It is reasonable to wonder if it is possible to weaken the hypothesis in Theorem 1.2 in a similar manner. In this paper, we give a new characterization of real hypersurfaces of type $A$ as motivated by the theorem given above, but instead of assuming that $M$ is Hopf, we assume that the distribution $\mathrm{D}$ is invariant under the shape operator $A$. We proved the following theorem:

Theorem 1.5. Let $M$ be a connected orientable real hypersurface in $G_{2}\left(C^{m+2}\right), m \geq 3$. If $A \mathrm{D} \subset \mathrm{D}$ and $\xi \in \mathrm{D}^{\perp}$, then $M$ is Hopf.

By Theorem 1.2 and Theorem 1.5, we have:

Theorem 1.6. Let $M$ be a connected orientable real hypersurface in $G_{2}\left(C^{m+2}\right), m \geq 3$. If $A \mathrm{D} \subset \mathrm{D}$ and $\xi \in \mathrm{D}^{\perp}$, then $M$ is an open part of a tube around a totally geodesic $G_{2}\left(C^{m+1}\right)$ in $G_{2}\left(C^{m+2}\right)$.

\section{REAL HYPERSURFACES IN $G_{2}\left(C^{m+2}\right)$}

In this section, we summarize and list out some important formulae as well as well-known results in the theory of real hypersurfaces in complex two-plane Grassmannians. We begin this section with the notations that we are going to use throughout this paper and also some basic properties of complex two-plane Grassmannians.

First, we denote the set of all complex 2-dimensional linear subspaces of $C^{m+2}$ by $G_{2}\left(C^{m+2}\right)$. The Riemannian metric is denoted by $g$. Next, we denote by $J$ and $J$ the Kähler structure and the quaternionic Kähler structure on $G_{2}\left(C^{m+2}\right)$ respectively. For each $x \in G_{2}\left(C^{m+2}\right)$, we denote by $\left\{J_{1}, J_{2}, J_{3}\right\}$ a canonical local basis of $\mathrm{J}$ on a neighborhood $\mathrm{U}$ of $x$ in $G_{2}\left(C^{m+2}\right)$, that is, each $J_{a}$ is a local almost Hermitian structure such that

$$
J_{a} J_{a+1}=J_{a+2}=-J_{a+1} J_{a}, \quad a \in\{1,2,3\} .
$$

The indices in the preceding equation is taken modulo three. The Levi-Civita connection of $G_{2}\left(C^{m+2}\right)$ is denoted by $\bar{\nabla}$. Since $\mathbf{J}$ is parallel with respect to the Levi-Civita connection $\bar{\nabla}$ of $G_{2}\left(C^{m+2}\right)$, for any canonical local basis $\left\{J_{1}, J_{2}, J_{3}\right\}$ of $\mathbf{J}$, there exist three local 1 -forms $q_{1}, q_{2}, q_{3}$ such that

$$
\bar{\nabla}_{X} J_{a}=q_{a+2}(X) J_{a+1}-q_{a+1}(X) J_{a+2}
$$

for each vecor field $X$ on $G_{2}\left(C^{m+2}\right)$, where $\bar{\nabla}$ is the Levi-Civita connection on $G_{2}\left(C^{m+2}\right)$. The Kähler structure $J$ and the quaternionic Kähler structure $\mathbf{J}$ are related by

$$
J_{a}=J_{a} J \quad \text { and } \quad \operatorname{Trace}\left(J J_{a}\right)=0,
$$

for all $a \in\{1,2,3\}$. Note that all the indices are taken modulo three. Next, the Riemannian curvature tensor $\bar{R}$ of $G_{2}\left(C^{m+2}\right)$ is locally given by 


$$
\begin{aligned}
\bar{R}(X, Y) Z= & g(Y, Z) X-g(X, Z) Y \\
& +g(J Y, Z) J X-g(J X, Z) J Y-2 g(J X, Y) J Z \\
& +\sum_{a=1}^{3}\left\{g\left(J_{a} Y, Z\right) J_{a} X-g\left(J_{a} X, Z\right) J_{a} Y-2 g\left(J_{a} X, Y\right) J_{a} Z\right. \\
& \left.+g\left(J J_{a} Y, Z\right) J J_{a} X-g\left(J J_{a} X, Z\right) J J_{a} Y\right\},
\end{aligned}
$$

for all $X, Y, Z \in T_{x}\left(G_{2}\left(C^{m+2}\right)\right)$.

For a nonzero vector $X \in T_{x}\left(G_{2}\left(C^{m+2}\right)\right)$, we denote by $C X=\operatorname{span}\{X, J X\}, \quad \mathbf{J} X=\left\{J^{\prime} X \mid J^{\prime} \in \mathbf{J}{ }_{x}\right\}$, $\mathrm{H} X=\operatorname{span}\{X\} \oplus \mathbf{J} X$, and $H C X$ the subspace spanned by $\mathrm{H} X$ and $\mathrm{H} J X$. If $J X \in \mathrm{J} X$, we denote by $C^{\perp} X$ the orthogonal complement of $C X$ in $\mathrm{H} X$.

Let $M$ be a connected, oriented real hypersurface isometrically immersed in $G_{2}\left(C^{m+2}\right), m \geq 3$, and $N$ a unit normal vector field on $M$. The Riemannian metric on $M$ will also be denoted by $g$ as there will be no confusion occured. A canonical local basis $\left\{J_{1}, J_{2}, J_{3}\right\}$ of $\mathrm{J}$ on $G_{2}\left(C^{m+2}\right)$ induces a local almost contact metric 3-structure $\left(\phi_{a}, \xi_{a}, \eta_{a}, g\right)$ on $M$ by

$$
J_{a} X=\phi_{a} X+\eta_{a}(X) N, \quad J_{a} N=-\xi_{a}, \quad \eta_{a}(X)=g\left(X, \xi_{a}\right)
$$

for all vector field $X$ tangent to $M$. It follows that

$$
\begin{aligned}
& \phi_{a} \phi_{a+1}-\xi_{a} \otimes \eta_{a+1}=\phi_{a+2}=-\phi_{a+1} \phi_{a}+\xi_{a+1} \otimes \eta_{a} \\
& \phi_{a} \xi_{a+1}=\xi_{a+2}=-\phi_{a+1} \xi_{a} .
\end{aligned}
$$

Let $(\phi, \xi, \eta, g)$ be the almost contact metric structure on $M$ induced by $J$, that is,

$$
J X=\phi X+\eta(X) N, \quad J N=-\xi, \quad \eta(X)=g(X, \xi)
$$

A real hypersurface $M$ is said to be Hopf if the Reeb vector field $\xi$ is principal.

It follows from (1) that the two structures $(\phi, \xi, \eta, g)$ and $\left(\phi_{a}, \xi_{a}, \eta_{a}, g\right)$ are related as follows

$$
\phi_{a} \phi-\xi_{a} \otimes \eta=\phi \phi_{a}-\xi \otimes \eta_{a} ; \quad \phi \xi_{a}=\phi_{a} \xi
$$

Next, we denote by $\nabla$ the Levi-Civita connection and $A$ the shape operator on $M$. Then

$$
\begin{aligned}
& \left(\nabla_{X} \phi\right) Y=\eta(Y) A X-g(A X, Y) \xi, \quad \nabla_{X} \xi=\phi A X, \\
& \left(\nabla_{X} \phi_{a}\right) Y=\eta_{a}(Y) A X-g(A X, Y) \xi_{a}+q_{a+2}(X) \phi_{a+1} Y-q_{a+1}(X) \phi_{a+2} Y, \\
& \nabla_{X} \xi_{a}=\phi_{a} A X+q_{a+2}(X) \xi_{a+1}-q_{a+1}(X) \xi_{a+2},
\end{aligned}
$$

for any $X, Y$ tangent to $M$.

Finally we state some well-known results.

Lemma 2.1. ([4]) Let $M$ be a real hypersurface in $G_{2}\left(C^{m+2}\right), m \geq 3$. If $\xi$ is tangent to $\mathrm{D}$, then $A \phi \xi_{a}=0$, for $a \in\{1,2,3\}$.

Theorem 2.2. ([2]) Let $M$ be a real hypersurface of type $A$ in $G_{2}\left(C^{m+2}\right), m \geq 3$. Then $\xi \in \mathrm{D}^{\perp}$ at each point of $M$. Suppose $J_{1} \in \mathrm{J}$ such that $J_{1} N=J N$. Then $M$ has three (if $r=\pi / 2 \sqrt{8}$ ) or four (otherwise) distinct constant principal curvatures 


$$
\alpha=\sqrt{8} \cot (\sqrt{8} r), \quad \beta=\sqrt{2} \cot (\sqrt{2} r), \quad \lambda=-\sqrt{2} \tan (\sqrt{2} r), \quad \mu=0
$$

with some $r \in] 0, \pi / \sqrt{8}[$. The corresponding multiplicities are

$$
m(\alpha)=1, \quad m(\beta)=2, \quad m(\lambda)=2 m-2=m(\mu)
$$

and the corresponding eigenspaces are

$$
\begin{gathered}
T_{\alpha}=\operatorname{span}\{\xi\} \\
T_{\beta}=C^{\perp} \xi \\
T_{\lambda}=\left\{X: X \perp H \xi, J X=J_{1} X\right\} \\
T_{\mu}=\left\{X: X \perp H \xi, J X=-J_{1} X\right\}
\end{gathered}
$$

Next, we give a short treatment on a symmetric tensor field $\theta_{a}$ introduced in [4], which is essential in the proof of our main theorem. Let $M$ be a real hypersurface in $G_{2}\left(C^{m+2}\right), m \geq 3$. Corresponding to each canonical local basis $\left\{J_{1}, J_{2}, J_{3}\right\}$ of $\mathbf{J}$, we define a local endomorphism $\theta_{a}$ on $T M$ by

$$
\theta_{a} X:=\tan \left(J J_{a}\right)=\phi_{a} \phi X-\eta(X) \xi_{a}=\phi \phi_{a} X-\eta_{a}(X) \xi
$$

It follows from (2) that the equation of Gauss is given by

$$
\begin{aligned}
R(X, Y) Z= & g(Y, Z) X-g(X, Z) Y+g(A Y, Z) A X-g(A X, Z) A Y \\
& +g(\phi Y, Z) \phi X-g(\phi X, Z) \phi Y-2 g(\phi X, Y) \phi Z \\
& +\sum_{a=1}^{3}\left\{g\left(\phi_{a} Y, Z\right) \phi_{a} X-g\left(\phi_{a} X, Z\right) \phi_{a} Y-2 g\left(\phi_{a} X, Y\right) \phi_{a} Z\right. \\
& \left.+g\left(\theta_{a} Y, Z\right) \theta_{a} X-g\left(\theta_{a} X, Z\right) \theta_{a} Y\right\} .
\end{aligned}
$$

Similarly, by adopting the symmetric tensor field $\theta_{a}$, the Codazzi equation is given by

$$
\begin{aligned}
\left(\nabla_{X} A\right) Y-\left(\nabla_{Y} A\right) X= & \eta(X) \phi Y-\eta(Y) \phi X-2 g(\phi X, Y) \xi \\
& +\sum_{a=1}^{3}\left(\eta_{a}(X) \phi_{a} Y-\eta_{a}(Y) \phi_{a} X-2 g\left(\phi_{a} X, Y\right) \xi_{a}\right. \\
& \left.+\eta_{a}(\phi X) \theta_{a} Y-\eta_{a}(\phi Y) \theta_{a} X\right) .
\end{aligned}
$$

For each $x \in M$, we define a subspace $\mathrm{H}^{\perp}$ of $T_{x} M$ by

$$
\mathrm{H}^{\perp}:=\operatorname{span}\left\{\xi, \xi_{1}, \xi_{2}, \xi_{3}, \phi \xi_{1}, \phi \xi_{2}, \phi \xi_{3}\right\}
$$

Let $\mathrm{H}$ be the orthogonal complement of $H C \xi$ in $T_{x}\left(G_{2}\left(C^{m+2}\right)\right)$. Then, $T_{x} M=\mathrm{H} \oplus \mathrm{H}^{\perp}$ and $\mathrm{H}$ is invariant under $\phi, \phi_{a}$ and $\theta_{a}$. Moreover, $\theta_{a \mid \mathrm{H}}$ has two eigenvalues: 1 and -1 . The following lemmas summarize some important properties and facts about $\theta_{a}$ :

Lemma 2.4. ([4])

(a) $\theta_{a}$ is symmetric,

(b) $\operatorname{Trace}\left(\theta_{a}\right)=\eta\left(\xi_{a}\right)$, 
(c) $\theta_{a}^{2} X=X-g\left(X, \phi \xi_{a}\right) \phi \xi_{a}$, for all $X \in T M$,

(d) $\theta_{a} \xi=-\xi_{a} ; \quad \theta_{a} \xi_{a}=-\xi ; \quad \theta_{a} \phi \xi_{a}=\eta\left(\xi_{a}\right) \phi \xi_{a}$,

(e) $\theta_{a} \xi_{a+1}=\phi \xi_{a+2}=-\theta_{a+1} \xi_{a}$,

(f) $\theta_{a} \phi \xi_{a+1}=-\xi_{a+2}+\eta\left(\xi_{a+1}\right) \phi \xi_{a}$,

(g) $\theta_{a+1} \phi \xi_{a}=\xi_{a+2}+\eta\left(\xi_{a}\right) \phi \xi_{a+1}$.

Lemma 2.5. ([4]) Let $\mathrm{H}_{a}(\varepsilon)$ be the eigenspace corresponding to eigenvalue $\varepsilon$ of $\theta_{a \mid \mathrm{H}}$. Then

(a) $\theta_{a \mid \mathrm{H}}$ has two eigenvalues $\varepsilon= \pm 1$,

(b) $\phi \mathrm{H}_{a}(\varepsilon)=\mathrm{H}_{a}(\varepsilon)$,

(c) $\theta_{b} \mathrm{H}_{a}(\varepsilon)=\mathrm{H}_{a}(-\varepsilon)$, for $a \neq b$,

(d) $\operatorname{dim}_{a}(1)=\operatorname{dim}_{a}(-1)$ is even,

(e) $\phi_{b} \mathrm{H}_{a}(\varepsilon)=\mathrm{H}_{a}(-\varepsilon)$, for $a \neq b$.

Note that $\xi \in \mathrm{D}^{\perp}$ if and only if $\operatorname{dim} \mathrm{H}^{\perp}=3$, that is, when $\xi \in \mathrm{D}^{\perp}$, we have $\mathrm{H}^{\perp}=\mathrm{D}^{\perp}$. One may refer to [4] for more details.

\section{PROOF OF THEOREM 1.5}

The assumption that $A D \subset D$ also implies $A D^{\perp} \subset D^{\perp}$. Hence, by a suitable choice of canonical local basis $\left\{J_{1}, J_{2}, J_{3}\right\}$ for $\mathbf{J}$, the vector fields $\xi_{1}, \xi_{2}, \xi_{3}$ are principal everywhere, say $A \xi_{a}=\beta_{a} \xi_{a}$, for $a=1,2,3$.

From the Codazzi equation (3), we have:

$$
\begin{aligned}
g\left(\left(\nabla_{X} A\right) Y-\left(\nabla_{Y} A\right) X, \xi_{a}\right) & =-2 \eta\left(\xi_{a}\right) g(\phi X, Y)-2 g\left(\phi_{a} X, Y\right) \\
& +2 \eta(X) \eta_{a}(\phi Y)-2 \eta(Y) \eta_{a}(\phi X) \\
& +2 \eta_{a+1}(X) \eta_{a+2}(Y)-2 \eta_{a+1}(Y) \eta_{a+2}(X) \\
& +2 \eta_{a+1}(\phi X) \eta_{a+2}(\phi Y)-2 \eta_{a+1}(\phi Y) \eta_{a+2}(\phi X),
\end{aligned}
$$

for all vector fields $X, Y$ tangent to $M$. On the other hand, since the shape operator $A$ is symmetric, $\nabla_{X} A$ is also symmetric, that is, $g\left(\left(\nabla_{X} A\right) Y, Z\right)=g\left(Y,\left(\nabla_{X} A\right) Z\right)$. Due to the fact that the canonical local basis is chosen in a way that $\xi_{a}, a=1,2,3$ are principal, we have

$$
\begin{aligned}
g\left(\left(\nabla_{X} A\right) Y-\left(\nabla_{Y} A\right) X, \xi_{a}\right)= & g\left(\left(\nabla_{X} A\right) \xi_{a}, Y\right)-g\left(\left(\nabla_{Y} A\right) \xi_{a}, X\right) \\
= & X\left(\beta_{a}\right) \eta_{a}(Y)-Y\left(\beta_{a}\right) \eta_{a}(X) \\
& +\beta_{a} g\left(\left(\phi_{a} A+A \phi_{a}\right) X, Y\right)-2 g\left(A \phi_{a} A X, Y\right) \\
& +\left(\beta_{a}-\beta_{a+1}\right)\left[q_{a+2}(X) \eta_{a+1}(Y)-q_{a+2}(Y) \eta_{a+1}(X)\right] \\
& -\left(\beta_{a}-\beta_{a+2}\right)\left[q_{a+1}(X) \eta_{a+2}(Y)-q_{a+1}(Y) \eta_{a+2}(X)\right] .
\end{aligned}
$$

Combining these equations, we have

$$
\begin{aligned}
& -2 \eta\left(\xi_{a}\right) g(\phi X, Y)-2 g\left(\phi_{a} X, Y\right)+2 \eta(X) \eta_{a}(\phi Y)-2 \eta(Y) \eta_{a}(\phi X) \\
& +2 \eta_{a+1}(X) \eta_{a+2}(Y)-2 \eta_{a+1}(Y) \eta_{a+2}(X)+2 \eta_{a+1}(\phi X) \eta_{a+2}(\phi Y)-2 \eta_{a+1}(\phi Y) \eta_{a+2}(\phi X) \\
& =X\left(\beta_{a}\right) \eta_{a}(Y)-Y\left(\beta_{a}\right) \eta_{a}(X)+\beta_{a} g\left(\left(\phi_{a} A+A \phi_{a}\right) X, Y\right)-2 g\left(A \phi_{a} A X, Y\right) \\
& +\left(\beta_{a}-\beta_{a+1}\right)\left[q_{a+2}(X) \eta_{a+1}(Y)-q_{a+2}(Y) \eta_{a+1}(X)\right] \\
& -\left(\beta_{a}-\beta_{a+2}\right)\left[q_{a+1}(X) \eta_{a+2}(Y)-q_{a+1}(Y) \eta_{a+2}(X)\right] .
\end{aligned}
$$


By taking $Y=\xi_{a}$, we obtain

$$
\begin{aligned}
X\left(\beta_{a}\right)= & \xi_{a}\left(\beta_{a}\right) \eta_{a}(X)-4 \eta\left(\xi_{a}\right) \eta_{a}(\phi X)+2 \eta\left(\xi_{a+1}\right) \eta_{a+1}(\phi X)+2 \eta\left(\xi_{a+2}\right) \eta_{a+2}(\phi X) \\
& +\left(\beta_{a}-\beta_{a+1}\right) q_{a+2}\left(\xi_{a}\right) \eta_{a+1}(X)-\left(\beta_{a}-\beta_{a+2}\right) q_{a+1}\left(\xi_{a}\right) \eta_{a+2}(X) .
\end{aligned}
$$

$Y\left(\beta_{a}\right)$ could be obtained in a similar manner. Some of these computations can be found in [2]. The following lemma is obtained by substituting both $X\left(\beta_{a}\right)$ and $Y\left(\beta_{a}\right)$ back into (4).

Lemma 3.1. Let $M$ be a connected orientable hypersurface in $G_{2}\left(C^{m+2}\right), m \geq 3$. Suppose $A \xi_{a}=\beta_{a} \xi_{a}$, for $a=1,2,3$, then

$$
\begin{aligned}
& 2 \eta\left(\xi_{a}\right) g(\phi X, Y)+2 g\left(\phi_{a} X, Y\right)+\beta_{a} g\left(\left(\phi_{a} A+A \phi_{a}\right) X, Y\right)-2 g\left(A \phi_{a} A X, Y\right) \\
& =+2 \eta(X) \eta_{a}(\phi Y)-2 \eta(Y) \eta_{a}(\phi X) \\
& +2 \eta_{a+1}(X) \eta_{a+2}(Y)-2 \eta_{a+1}(Y) \eta_{a+2}(X)+2 \eta_{a+1}(\phi X) \eta_{a+2}(\phi Y)-2 \eta_{a+1}(\phi Y) \eta_{a+2}(\phi X) \\
& +2 \eta_{a}(Y)\left[2 \eta\left(\xi_{a}\right) \eta_{a}(\phi X)-\eta\left(\xi_{a+1}\right) \eta_{a+1}(\phi X)-\eta\left(\xi_{a+2}\right) \eta_{a+2}(\phi X)\right] \\
& -2 \eta_{a}(X)\left[2 \eta\left(\xi_{a}\right) \eta_{a}(\phi Y)-\eta\left(\xi_{a+1}\right) \eta_{a+1}(\phi Y)-\eta\left(\xi_{a+2}\right) \eta_{a+2}(\phi Y)\right] \\
& +\left(\beta_{a}-\beta_{a+1}\right) q_{a+2}\left(\xi_{a}\right)\left[\eta_{a}(X) \eta_{a+1}(Y)-\eta_{a+1}(X) \eta_{a}(Y)\right] \\
& +\left(\beta_{a}-\beta_{a+2}\right) q_{a+1}\left(\xi_{a}\right)\left[\eta_{a+2}(X) \eta_{a}(Y)-\eta_{a}(X) \eta_{a+2}(Y)\right] \\
& -\left(\beta_{a}-\beta_{a+1}\right)\left[q_{a+2}(X) \eta_{a+1}(Y)-q_{a+2}(Y) \eta_{a+1}(X)\right] \\
& +\left(\beta_{a}-\beta_{a+2}\right)\left[q_{a+1}(X) \eta_{a+2}(Y)-q_{a+1}(Y) \eta_{a+2}(X)\right]
\end{aligned}
$$

for all $X, Y$ tangent to $M$.

We further assume that $\xi \in \mathrm{D}^{\perp}$. For any $X \in \mathrm{D}$, since $\mathrm{D}$ is invariant under $A, \phi$ and $\phi_{a}$, from equation (5), we have

$$
\begin{aligned}
0= & 2 \eta\left(\xi_{a}\right) g(\phi X, Y)+2 g\left(\phi_{a} X, Y\right)+\beta_{a} g\left(\left(\phi_{a} A+A \phi_{a}\right) X, Y\right)-2 g\left(A \phi_{a} A X, Y\right) \\
& -\left(\beta_{a}-\beta_{a+1}\right) q_{a+2}(X) \eta_{a+1}(Y)+\left(\beta_{a}-\beta_{a+2}\right) q_{a+1}(X) \eta_{a+2}(Y),
\end{aligned}
$$

for all $Y$ tangent to $M$ and $a=1,2,3$. Hence, we have

Lemma 3.2. Let $M$ be a connected orientable hypersurface in $G_{2}\left(C^{m+2}\right), m \geq 3$. Suppose $A \mathrm{D}^{\perp} \subset \mathrm{D}^{\perp}$ and $\xi \in \mathrm{D}^{\perp}$, if $A \xi_{a}=\beta_{a} \xi_{a}$, for $a=1,2,3$, then

$$
2 \eta\left(\xi_{a}\right) \phi X+2 \phi_{a} X+\beta_{a}\left(\phi_{a} A+A \phi_{a}\right) X-2 A \phi_{a} A X=0
$$

for all $X \in \mathrm{D}$.

Lemma 3.3. Let $M$ be a connected orientable hypersurface in $G_{2}\left(C^{m+2}\right), m \geq 3$. Suppose $A \mathrm{D}^{\perp} \subset \mathrm{D}^{\perp}$ and $\xi \in \mathrm{D}^{\perp}$, if $A \xi_{a}=\beta_{a} \xi_{a}$ for $a=1,2,3$, then for all $X \in \mathrm{D}$, we have $\phi_{a} A \phi_{a} A X=A \phi_{a} A \phi_{a} X$.

Proof. Note that if $X \in \mathrm{D}$, then $\phi_{a} X \in \mathrm{D}$. Next, applying $\phi_{a}$ on both sides of (7) and replacing $X$ by $\phi_{a} X$ in (7) give

$$
2 \eta\left(\xi_{a}\right) \theta_{a} X-2 X-\beta_{a} A X+\beta_{a} \phi_{a} A \phi_{a} X-2 \phi_{a} A \phi_{a} A X=0
$$


and

$$
2 \eta\left(\xi_{a}\right) \theta_{a} X-2 X+\beta_{a} \phi_{a} A \phi_{a} X-\beta_{a} A X-2 A \phi_{a} A \phi_{a} X=0
$$

respectively. Hence, $\phi_{a} A \phi_{a} A X=A \phi_{a} A \phi_{a} X$, for all $X \in \mathrm{D}$.

With the assumption that $\xi \in \mathrm{D}^{\perp}$, we have $\mathrm{D}=\mathrm{H}$ and $A \mathrm{H} \subset \mathrm{H}$. By Lemma 3.3, there exist common orthonormal eigenvectors $X_{1}, \cdots, X_{4 m-4} \in \mathrm{H}$ of $A$ and $\phi_{1} A \phi_{1}$. It follows that

$$
A X_{j}=\lambda_{j} X_{j}, \quad \text { and } \quad A \phi_{1} X_{j}=\mu_{j} \phi_{1} X_{j} .
$$

Using these in (7), we have

$$
2 \eta\left(\xi_{1}\right) \phi X_{j}+\left(2+\lambda_{j} \beta_{1}+\mu_{j} \beta_{1}-2 \lambda_{j} \mu_{j}\right) \phi_{1} X_{j}=0
$$

Since $\xi \in \mathrm{D}^{\perp}$, we may suppose $\eta\left(\xi_{1}\right) \neq 0$. Then

$$
0=\phi X_{j}+\varepsilon \phi_{1} X_{j}
$$

where $\varepsilon=\frac{2+\beta_{1}\left(\lambda_{j}+\mu_{j}\right)-2 \lambda_{j} \mu_{j}}{2 \eta\left(\xi_{1}\right)}$. Taking $\phi_{1}$ on both sides of (9) gives

$$
0=\theta_{1} X_{j}-\varepsilon X_{j}
$$

By Lemma 2.5 (a), $\varepsilon \in\{1,-1\}$. Without loss of generality, we can assume that

$$
X_{1}, \cdots, X_{2 m-2} \in \mathrm{H}_{1}(1) \quad \text { and } \quad X_{2 m-1}, \cdots, X_{4 m-4} \in \mathrm{H}_{1}(-1) \text {, }
$$

where $H_{1}(1)$ and $H_{1}(-1)$ are the eigenspace of $\left.\theta_{1}\right|_{H}$ corresponding to the eigenvalue 1 and -1 respectively. Consequently, (8) implies

$$
A \mathrm{H}_{1}(1) \subset \mathrm{H}_{1}(1)
$$

and hence

$$
\phi_{2} A \phi_{2} \mathrm{H}_{1}(1) \subset \mathrm{H}_{1}(1)
$$

Thus, if we take $a=2$ in Lemma 3.3, then there exists orthonormal vectors $\bar{X}_{1}, \cdots, \bar{X}_{2 m-2} \in \mathrm{H}_{1}(1)$ such that

$$
A \bar{X}_{j}=\bar{\lambda}_{j} \bar{X}_{j} \quad \text { and } \quad A \phi_{2} \bar{X}_{j}=\bar{\mu}_{j} \phi_{2} \bar{X}_{j} .
$$

From (7), we have

$$
2 \eta\left(\xi_{2}\right) \phi \bar{X}_{j}+\left(2+\bar{\lambda}_{j} \beta_{2}+\bar{\mu}_{j} \beta_{2}-2 \bar{\lambda}_{j} \bar{\mu}_{j}\right) \phi_{2} \bar{X}_{j}=0
$$

Since $\bar{X}_{j} \in \mathrm{H}_{1}(1), \phi \bar{X}_{j} \in \mathrm{H}_{1}(1)$ and $\phi_{2} \bar{X}_{j} \in \mathrm{H}_{1}(-1)$, we have 


$$
\eta\left(\xi_{2}\right)=0
$$

In a similar manner, we obtain $\eta\left(\xi_{3}\right)=0$. Thus, we have $\eta\left(\xi_{1}\right)= \pm 1$ or $\xi= \pm \xi_{1}$. As a result, we have shown that $A \xi=\alpha \xi$ with $\alpha=\beta_{1}$. This completes the proof of Theorem 1.5.

\section{REFERENCES}

1. J. Berndt, Riemannian geometry of complex two-plane Grassmannians, Rend. Semin. Mat. Univ. Politec. Torino 55, 19-83 (1997).

2. J. Berndt and Y. J. Suh, Real hypersurfaces in complex two-plane Grassmannians, Monatsh. Math.127, 1-14 (1999).

3. H. Lee and Y. J. Suh, Real hypersurfaces of type $B$ in complex two-plane Grassmannians related to the Reeb vector, Bull. Korean Math. Soc. 47, 551-561 (2010).

4. T. H. Loo, Semi-parallel real hypersurfaces in complex two-plane Grassmannians, Differ. Geom. Appl. 24, 87-102 (2014).

5. Y. J. Suh, Real hypersurfaces in complex two-plane Grassmannians with parallel shape operator, Bull. Aust. Math. Soc. 67, 493-502 (2003). 